\title{
Inconsistencies in institutional review board decisions: A proposal to regulate the decision-making process
}

\author{
Ekmekci PE${ }^{1}$, Guner $\mathrm{MD}^{2}$ \\ TOBB ETU Medical School Department of History of Medicine and Ethics, Cankaya/Ankara, Turkey. \\ drpelifek@gmail.com
}

\begin{abstract}
Medical sciences and medical technologies are developing in an enormous pace. The number of multi-site and multi- cultural clinical trials is increasing in line with this development. Yet, the increase in number brought about a problem, namely that of inconsistencies in institutional review board (IRB) decisions. The efforts to develop a comprehensive and systematic framework for the decision-making procedure of IRB members were ineffective and ended up in form of lists containing general principles of research ethics. The increase in multi-site, multicultural clinical research together with the initiation of new institutional review boards in countries involved in these researches emphasize the importance of defining the sine qua non criteria for considering a proposed research study to be ethical, as well as for avoiding inconsistencies in institutional review board decisions. The aim of this study is to prepare a toolkit for IRB members to regulate the ethical decision-making process while minimizing inconsistencies in institutional review board decisions. The objective is to minimize the effect of subjective factors and to ensure that all issues in key international documents and guidelines are covered prior to the point of reaching a conclusion for the proposed research protocol. By providing a comprehensive review of the Declaration of Helsinki, Council For International Organizations of Medical Sciences Guidelines, International Conference on Harmonization Guidelines, Standards and Operational Guidance for Ethics Review of Health-related Research with Human Participants of World Health Organization, and Medical Ethics Manual of the World Medical Association, this study aims at developing a standard evaluation sheet for institutional review board members to evaluate the ethical appropriateness of proposed trial protocols (Tab. 1, Ref. 35). Text in PDF www.elis.sk.

KEY WORDS: clinical research, ethics review committee, human subjects, informed consent, institutional review board, research ethics.
\end{abstract}

List of abbreviations: CIOMS - The Council for International Organizations of Medical Sciences, COI - Conflict of interest, DoH - The Declaration of Helsinki, EGES - Ekmekci-Güner Ethical Evaluation Sheet, EU -The European Union, GCP - Good Clinical Practice, ICH-GCP - The International Conference on Harmonization-Good Clinical Practice, IRB - Institutional Review Boards, UNESCO - The United Nations Educational, Scientific, and Cultural Organization, USA - The United States of America, WHO World Health Organization, WMA - World Medical Association.

\section{Introduction}

Historic background of institutional review boards

The Nuremberg code published after the tragic events of World War II initiated incentives to protect the rights of human subjects in clinical research. However, the requirement of formal written

${ }^{1}$ TOBB ETU Medical School Department of History of Medicine and Ethics, Cankaya/Ankara, Turkey, and ${ }^{2}$ TOBB ETU Medical School Department of Clinical Pharmacology, Cankaya/Ankara, Turkey

Address for correspondence: P.E. Ekmeci, TOBB ETU Medical School Department of History of Medicine and Ethics, Sogutozu Mahallesi, Sogutozu Cd. No: 43, 06510 Cankaya/Ankara, Turkey. ethical approval from an independent committee became mandatory as late as in 1975 when the first revision of the Declaration of Helsinki was published (Carlson et al, 2004).The final revision of the Declaration of Helsinki was published in Fortaleza, Brazil, in 2013, and is today considered the gold standard for research on human subjects (WMA, 2013). International organizations have been established to unify the standards of good clinical research. The Council for International Organizations of Medical Sciences (CIOMS), a non-governmental, non-profit organization was jointly established by the World Health Organization (WHO) and United Nations Educational, Scientific and Cultural Organization (UNESCO) in 1949. CIOMS produced guidelines for ethical research conduct in 1993 and updated them in 2002. These guidelines mainly address cultural differences in multi-national studies. The International Conference on Harmonization - Good Clinical Practice (ICH-GCP), another international body composed of experts from the pharmaceutical industry and regulatory authorities from Japan, European Union (EU), United States of America (USA), Canada, Nordic countries, and WHO, published guidelines for Good Clinical Practice (GCP) to offer standardization for clinical drug trials (Kim, 2012).

The number of Institutional Review Boards (IRB) has substantially increased, especially in the USA and European countries, 
where the majority of clinical research is being conducted. In 2008, there were 3,853 IRBs reviewing more than a quarter-million research applications in the USA alone (Catania et al, 2008). According to the Office for Human Research Protections Database, the total number of IRBs in the USA is 5,934 and the total number of international IRBs is 4,998 (HHS - OHRP 2017). The increase in their number brought about a problem of inconsistencies in IRB decisions.

The increase in multi-site and multi-cultural clinical research, together with growing skepticism about the universality of the international guidelines urged scholars to define the sine qua non criteria for considering a proposed research to be ethical (Macklin, 2001, Emanuel et al, 2008). In this respect Emanuel et al offered eight principles and benchmarks including collaborative partnership, social value, scientific validity, fair participant selection, favorable risk-benefit ratio, independent review, informed consent and respect for participant (Emanuel, Wendler, Killen, \& Grady, 2004;Emanuel, 2000). The aim of this work was "to provide a comprehensive and systematic framework to guide the ethical conduct of clinical research and minimize the possibility of exploitation” (Emanuel et al, 2008, 2004, Emanuel, 2000) This work was valuable especially for the structure which "sequentially presented principles starting from the development of research proposals, through to the conduct of research and monitoring during research” (Emanuel et al, 2008.) However, this framework was a refined version of general principles of research ethics for all parties involved in clinical research on human subjects. It was not a tool developed in particular for decision making of IRB members. This situation led to inconsistent IRB decisions over the same study and thus grew into a pressing problem (Bledsoe et al, 2007, Emanuel et al, 2004).

A survey by Green et al shows how significant the variations in IRB decisions over the same study can be. In this study of 43 IRBs, ten decided in favor of expedited review, 31 provided a full board review, and one IRB objected to the study because of risks involved (Green et al, 2006). These inconsistencies urge investigators to question the validity and objectivity of the decisionmaking procedure in IRBs (Ashcraft and Krause, 2007, De Vries et al, 2004, Keith-Spiegel and Koocher, 2005). The Illinois white paper on improving the system for protecting human subjects states that "the greatest irony of the entire IRB debate is how little of it is informed by actual, reliable research about the facts of the problem ...Examination reveals that virtually no scientific evidence is brought to bear on any aspect of the debate about how IRBs function” (The Center for Advanced Study Project Steering Committee, 2005).

The aim of this study is to prepare a toolkit to regulate the ethical decision-making process for IRB members. The objective is to minimize the effect of subjective factors such as personal intuitions, feelings, beliefs and interpersonal interactions, irrational influences on group decision-making, and to ensure that all issues in key international documents and guidelines are covered prior to the point of reaching a conclusion on the proposed research protocol.

\section{Methodology}

Guidelines issued by institutions, namely the Declaration of Helsinki (DoH), World Medical Association (WMA, 2013), CIOMS guidelines (Organizations \& Sciences, 2016), ICH guidelines (ICH Harmonized Tripartite Guideline, 1996), Standards and Operational Guidance for Ethics Review of Health-Related Research with Human Participants of WHO (WHO, 2011), Medical Ethics Manual of the World Medical Association (Cataloguing-in-Publication Data, Williams \& John, 1942), Ethics Review Board Resources: Guidebook for Members of Ethics Review Board University of California (Harayil, 2005), and Boston Children's Hospital IRB Reviewer Worksheet New Protocols Full Review are reviewed and the ethical issues that should be considered during the decision-making process are identified. These documents were merged to form a single outline and a table of correspondence was

Tab. 1. Revision criteria for informed consent document*.

\begin{tabular}{|c|c|}
\hline Criteria & Items evaluated \\
\hline $\begin{array}{l}\text { Scientific design and conduct of the } \\
\text { clinical trial }\end{array}$ & $\begin{array}{l}\text { Research design and methodology, current supporting scientific literature, preliminary data, justification of } \\
\text { the study, qualifications of the investigators and institutions, expected contribution of the results, analysis } \\
\text { and publication plan, }\end{array}$ \\
\hline $\begin{array}{l}\text { Selection of the study population and } \\
\text { recruitment of research participants }\end{array}$ & $\begin{array}{l}\text { Selection criteria, sample size, recruitment methodology, gender equality, vulnerability status and special } \\
\text { protection requirement, social value of the research }\end{array}$ \\
\hline $\begin{array}{l}\text { Care and protection of research } \\
\text { subjects }\end{array}$ & $\begin{array}{l}\text { Possible risks, harms and benefits to study population, resources and precautions to minimize possible risks } \\
\text { and harms, post research provisions, equitable distribution of benefits and burdens, plan for interim analy- } \\
\text { sis, confidentiality precautions, and risks for vulnerable groups. Research on children, pregnant women or } \\
\text { fetuses, individuals in hierarchical relationships, institutionalized persons and non-therapeutic clinical re- } \\
\text { search on incompetent participants, and in low-resource settings }\end{array}$ \\
\hline Informed consent & $\begin{array}{l}\text { Compliance of the content of the document and procedure with the standards of international guidelines } \\
\text { and recommendations }\end{array}$ \\
\hline Issues related to conflict of interest & $\begin{array}{l}\text { The disclosure of conflict of interest of sponsor/principal investigator/researchers and presence of any pos- } \\
\text { sible adverse effects on research conduct, integrity and results }\end{array}$ \\
\hline $\begin{array}{l}\text { Inducements, financial benefits, and } \\
\text { financial costs: reimbursement and } \\
\text { compensation }\end{array}$ & $\begin{array}{l}\text { Definition and schedule of all compensations, payments and supplies; presence of coercive or attractive } \\
\text { payments or supplies }\end{array}$ \\
\hline
\end{tabular}

* EGES is provided as an addendum to this paper or will be provided to the interested professionals upon request by contacting the corresponding author via e-mail. 
prepared to see to it that the document outline was in compliance with the ethical requirements of the DoH. The final document is prepared by taking into account the table of correspondence and outline document.

\section{Results}

The revision criteria in the Ethical Evaluation Sheet (EGES) for clinical research protocols on human subjects consist of six essential parts as follows (Tab. 1):

1) Scientific design and conduct of the clinical trial

2) Selection of study population and recruitment of research participants

3) Care and protection of research subjects

4) Informed consent

5) Conflict of interest issues

6) Inducements, financial benefits, and financial costs; reimbursement and compensation

\section{Scientific design and conduct of the clinical trial}

\section{Risks related to research design}

This section starts with the essential qualification of the scientific value and soundness of the research. No research on human subjects should be conducted when these two qualifications are not met. In some countries, research proposals go through scientific evaluations before they are submitted to IRB. In this case, IRB can take the decision of scientific committees into account and approve the scientific soundness. However, it is important to remember that it is among the responsibilities of the IRB to decide on the scientific validity of the proposed research. The research protocol should provide relevant scientific data obtained from previous laboratory and animal experiments, and existing knowledge in the relevant academic literature to justify both the scientific and social value of the research.

The physical, human, and technological resources of the research team, and institution where the research is to be conducted should be declared. Additionally, the qualifications specified by the applicable regulatory requirement(s) and evidence of such qualifications through up-to-date curriculum vitae and/or other relevant documentation and their compliance with Good Clinical Practices, need to be provided.

All reasonably relevant physical, mental, social, and economic risks to the research subjects should be considered while providing enough evidence about the existence of adequate planning to minimize these risks.

The sample size and methodology should be evaluated and justified. Some IRBs require a statement that statistical appropriateness is approved by a competent statistician. However, similarly to the scientific committee approval, it is still the IRB's responsibility to assess these issues from a point of view that prioritizes the minimizing of any risk of harm for human subjects.

The research methodology should be scientifically proven and well defined. The differences between research protocol and standard operations should be made clear. If the methodology in- volves deception of subjects, this should be justified by ethical, legal, and scientific means, and the subjects should be informed that they may be deceived and that they can face additional risks of harm as a result.

The generalization of knowledge is the ethical and scientific responsibility of researchers. Therefore, both the registration of clinical research into a public database and the reassurance of IRB that negative and non-significant results will be made public are important.

\section{Selection of study population and recruitment of research participants}

The research protocol should explain why the proposed group of participants is the most favorable group for the research. If the research is to be conducted on people with vulnerabilities, then even stricter justification is required for approval. Apart from the profile of the participants, the total number of subjects and the inclusion and exclusion criteria should be defined and justified in ethical and scientific terms. Avoiding any type of discrimination, including gender and age, is very important to ameliorate the disadvantages of under-presented groups in clinical research; hence, the participant profile should be assessed from this point of view.

The location and setting of approaching a potential subject for recruitment should be well defined and justified so that it does not embrace any undue influence or coercion. The timing and setting should respect the privacy of the participants and provide them with enough time to ask questions and carefully consider all relevant aspects of participating in the research. The existing resources should be sufficient to enable researchers to recruit the proposed number of subjects and to complete the research in compliance with the research protocol.

The social value of the research should be assessed according to the research's direct relevance on the understanding or intervention of a significant health problem, and measure by which it contributes to the promotion of individual and public health.

\section{Care and protection of research subjects}

All reasonable and probable physical, mental, social, economic, and legal risks and benefits should be defined. No probable risks should be left out due to low magnitude. Legal risks should be considered in terms of local laws and regulations as well as universal human rights.

The allocation of risks and benefits among different groups should be taken into account. In some research, the burden of risk may fall on one group while the second group is only subject to the benefits. In other cases, the benefits may fall on both groups while only one group faces the risk of harm, or only one group is subject to both risks and benefits. The research protocol should be evaluated carefully from this point of view to ensure that the principle of justice is respected in risk benefit allocation.

Any research that is to be conducted on a vulnerable group should satisfy the following pre-conditions: 1) the research can- 
not be carried out with a non-vulnerable group, 2) the research is responsive to the health needs or priorities of this vulnerable group, and 3) this vulnerable group stands to benefit from the knowledge, practices, or interventions that result from the research.(WMA, 2013)

Research on particular vulnerable groups such as children, pregnant women or fetuses, individuals with low resources or those who are in hierarchical relationships with researchers, institutionalized people, and incompetent individuals should be considered with particular attention to avoid any breach of rights, disrespect for autonomy, and abuse.

Post-trial medical provisions are very important especially for subjects from low-resource communities. If the results prove that there is a scientifically substantial benefit for the subjects, there should be a plan in place to make the new intervention available to them after the research is finished.

The use of placebos or any intervention that is inferior to standard care is another issue that requires particular attention to avoid any ethical violations. The DoH CIOMS, and ICH guidelines underline that the benefits, risks, burdens, and effectiveness of new interventions should be tested against those of the best proven intervention. If the research design does not test the new intervention against the best proven intervention, then the reasons for such a design should be justified.

The scientific and administrative qualifications of those who are in charge of data monitoring, as well as mechanisms to stop or revise the research as soon as the monitored data show particular traits, should be specified. If personal data are to be collected, appropriate provisions to secure the privacy and confidentiality of participants should be taken. If the research design requires breaching the data confidentiality, this should be justified ethically, legally, and scientifically, and all measures to minimize the risk of harm to subjects should be taken.

In multi-site or multi-country research, the confidentiality and privacy of personal data should be considered with particular attention, since the fact that various people can be involved in the collection, transfer, and analysis of the data, increases the risk of breaches.

\section{Informed consent}

The evaluation of informed consent is focused on appropriateness of two main aspects, namely that of informed consent document and informed consent procedure. Written informed consent is the gold standard with respect to autonomy. However, some circumstances and subject profiles may make it impossible to acquire informed consent in written form. If written informed consent is not to be utilized, the explanation should be justified both ethically and legally. Adequate measures to respect the autonomy of the subjects should be taken and written down in the research protocol.

The informed consent document allows the subjects to decide whether or not they would like to opt out of the research. Therefore, it should be carefully examined to ensure that it contains all the relevant information so that the subject is free from undue in- fluence, coercion, or pressure. The informed consent form should include the items in Table 1.

\section{The content of the informed consent document}

The second part of this section focuses on the assessment of the informed consent procedure. Although drawing up the informed consent document so that it includes all the relevant information showed in Table 1 is important, it is only the first step of the informed consent procedure. The whole procedure includes all the steps, starting from approaching the potential participant and asking for permission, through to talking about the clinical research that the potential participant might be interested in. The process includes identifying the person who is to complete the informed consent document, defining his/her qualifications (including communication and language skills), and time allocated for answering questions as well as that for subjects to think through and discuss their participation with their relatives. Particular measures should be taken for subjects with little or no competency in decisionmaking regarding advance directives, surrogate decision-making, and patients' best interest.

Using residual tissue from clinical diagnosis or treatments in clinical research is not exempt from informed consent. Any research that is going to use residual tissue should explain how informed consent is to be achieved.

\section{Conflict of interest (COI)}

COI is an issue that is frequently under evaluation by IRBs. The declaration of COI by the sponsor, principle investigator, or researchers should be assessed regarding their influence on the protection of subjects, recruitment procedures, equitable and unbiased selection and grouping of subjects, as well as on research integrity.

\section{Inducements, financial benefits, and financial costs; reim- bursement and compensation}

Incentives such as coercive payments or provision of supplies that are difficult for potential participants to refuse, cannot be justified ethically. The schedule of payment is also very important and is usually underestimated. IRB should make sure that the course of payments does not urge the subjects to continue with the research even if they think that it is best for them to withdraw.

\section{Discussion}

\section{A common problem of inconsistencies in IRB decisions}

In his article on the decision-making procedures of IRBs, Pritchard, lists the reasons for inconsistencies in IRB decisions as follows (Pritchard, 2011):

1) Variations emerging from religious, cultural, and ethnic backgrounds not only affect the ethical values, they also alter the decision-making process. In Western countries the decisionmaking process is individualistic, whereas in the Eastern world, it is considered to be communitarian. 
2) The vast majority of changes in the research environment, and IRB members' lack of familiarity relevant to the assessment of a proposed study: The IRB members may not be familiar with the existing literature and medical interventions in the relevant field. Failing to consult with an expert in the area might lead to wrong decisions.

3) The IRBs variate in their application of regulations on clinical research, either because of insufficient comprehension of regulations or because the regulations themselves are not clear.

4) The psychological patterns of human beings in their individual judgments and decision-making: IRB members may be influenced by the group decision-making process.

The list of factors jeopardizing the effectiveness and validity of IRB decisions is widened by Brown by adding "the pressure of reviewing too much, too little, with too little expertise and training, struggling with conflicts threatening the independence of IRB, and lack of evaluation and oversight of IRB decisions and functioning” (Brown, 1998).

Some researchers look for the sources of inconsistent decisions in psychologically oriented factors such as relationships between members' roles on IRB, agreement with other members or previous decisions without conducting an extensive assessment of risks, benefits, and scientific facts, and effect of authority of some members (Pritchard, 2011). Others focus on personal dimensions of risk-benefit assessments and argue that IRB members evaluate risk and benefit based on intuition, impressions, or personal experience rather than on objective scientific data (Van Luijn et al, 2002, Stark and Schrag, 2012).

The idea of developing the EGES emerged from the need to make the decision-making process as rational as possible, stripped from cultural and religious subjectivities, and immune to psychological patterns of group decision-making. The EGES goes through the important ethical and scientific issues related to research with IRB member, so that the decision criterion rests on these facts rather than on subjective influences.

The ethical and scientific criteria are carefully reviewed and listed in accordance with internationally accepted documents. Two issues of CIOMS guidelines are deliberately excluded from EGES, namely 1) using residual tissue without informed consent; and 2) research that includes sham surgical procedures.

For the case of using residual tissue without informed consent, CIOMS guideline11 on collection, storage, and use of biological materials and related data states as follows:

"When human biological materials are left over after clinical diagnosis or treatment (so-called "residual tissue") and are stored for future research, a specific or broad informed consent may be used or substituted by an informed opt-out procedure. This means that the material is stored and used for research unless the person from whom it originates explicitly objects. The informed opt-out procedure must fulfil the conditions as follows: 1) patients need to be aware of its existence, 2) sufficient information needs to be provided, 3) patients need to be told that they can withdraw their data, and 4) a genuine possibility to object has to be offered”(Organizations \& Sciences, 2016).

Therefore, this includes the requirements for opt-out proce- dure, it emphasizes that patients should be made aware of the existence of such a process, their right to withdraw data, and offering them a genuine possibility to object. This is in accordance with ethical principles for respecting autonomy, protection of privacy and confidentiality, and honesty. However, the following statement of CIOMS guideline 11 is quite problematic in terms of ethical principles:

"When researchers seek to use stored materials collected for past research, or clinical or other purposes without having obtained informed consent for their future use for research, the research ethics committee may waive the requirement of individual informed consent if 1 ) the research would not be feasible or practicable to carry out without the waiver, 2) the research has important social value, and 3) the research poses no more than minimal risks to participants or group to which the participant belongs” (Organizations \& Sciences, 2016).

Acquiring informed consent for the use of residual tissue or stored materials in clinical research has always been a major problem. This problem has reached a new dimension with the development of biobanks (McGuire and Beskow, 2010). The biological specimens that are collected and stored at these institutions constitute a tremendous pool of material for clinical research. In most cases, acquiring informed consent for the use of stored material is difficult and time-consuming. It is easier for researchers to claim that "the research would not be feasible or practicable to carry out without the waiver." However, several mechanisms have been developed for an ethically approved informed consent procedure that does not breach the respect for autonomy and privacy of participants. This paragraph from the CIOMS Guideline 11 might easily be misused by creating a fabricated excuse for researchers to waive informed consent. In addition, there is a lack of consensus about this problematic issue in various international guidelines which contain fundamentally different approaches (Elger and Caplan, 2006).

The requirement for justifying the social value of the research is the basic ethical requirement for all clinical research on human subjects, and it is irrelevant to waiving the informed consent. Therefore, this statement from CIOMS is not included in EGES.

Research that includes sham surgical procedures:

Beecher described the placebo effect of surgery after conducting a randomized controlled trial of internal mammary artery ligation against a sham surgery for angina pectoris in 1959 (Cobb et al, 1959). Since then, the use of sham surgical procedures in clinical research on human subjects has been debated.

The risk of harm due to anesthesia, skin incisions, and other invasive interventions related to sham surgeries make it very difficult to comply with the basic ethical principles of doing no harm. This statement may be contradicted since the entire clinical research involves some risk of harm, and risk of harm is acceptable as long as the expected benefits override the risks. However, in the case of using placebos in clinical research, the DoH is very clear about balancing the risks and benefits. The Article 33 of DoH on use of placebos states as follows:

"Where no proven intervention exists, the use of placebo, or no intervention, is acceptable; or where for compelling and scien- 
tifically sound methodological reasons the use of any intervention less effective than the best proven one, the use of placebo, or no intervention is necessary to determine the efficacy or safety of an intervention and the patients who receive any intervention less effective than the best proven one, placebo, or no intervention will not be subject to additional risks of serious or irreversible harm as a result of not receiving the best proven intervention. Extreme care must be taken to avoid abuse of this option” (WMA, 2013).

The approach in the CIOMS guidelines for sham surgery is as follows:

"In some fields such as surgery and anesthesia, testing the effectiveness of interventions may require the use of sham interventions. For example, the participants in the active arm of a surgery trial may receive arthroscopic surgery on their knees, while participants in the control group may receive only a minor skin incision. In other cases, both groups may receive an invasive procedure such as inserting a catheter into a person's artery. The catheter is threaded into the heart of participants in the active arm but stopped short of the heart in participants in the control arm. The risks of sham procedures can be considerable (for example, surgical incision under general anesthesia) and must be carefully considered by a research ethics committee" (Organizations \& Sciences, 2016).

This is not in compliance with DoH, since it approves the use of placebos (sham surgeries) where proven interventions exist, and the sham intervention will be subject to additional risks of serious or irreversible harm as a result of the participants not receiving the best proven intervention (WMA, 2013).

Today, the dilemma about sham surgery is still unsolved. On the one hand, the requirements for the use of sham surgery in clinical research in neurological sciences, orthopedics, and cardiovascular surgery is increasing (Freeman et al, 1999, Dekkers and Boer, 2001, Wolf and Buckwalter, 2006), on the other hand, discussions on the ethical acceptability of the high risk of harm, sacrificing the best interests of participants for the sake of generating new knowledge, and ignoring the duties of physicians have also increased (Hostiuc et al, 2016, Emanuel and Miller, 2001, Horng and Miller, 2002, London and Kadane, 2003, Miller and Kaptchuk, 2004). The lack of consensus among parities as well as lack of compliance between the CIOMS guidelines and DoH have led us to exclude the use of sham surgery in clinical research from EGES. It may be included in the future after we reach more clarity and consensus.

\section{What is not covered in EGES?}

The EGES does not cover particular types of research such as research of disasters and disease outbreaks, cluster randomized trials, research on data obtained from online environment, and digital tools used in health-related research. These clearly require an additional evaluation criterion for IRB approval. Annexes that are devoted to the evaluation of these and other newly emerging types and areas of research are needed.

\section{Need for revision due to national laws and regulations}

The EGES does not include any national regulations for the purpose of serving as a universal guide for all IRBs around the world. However, it is a fact that an IRB member has to consider the national regulations of the country before reaching the judgement about the research protocol. Therefore, a phrase relating to "taking into account local laws and regulations" is added to every sentence where legal requirements are assessed. Hence, for practical purposes, the EGES should be reviewed and the national legal requirements should be added to the document before putting it to use in any country. The document should be revised to address issues such as the requirement for a scientific committee review prior to IRB review, mandatory insurance for all participants, allowing research on leftover embryos from assisted reproductive techniques, and so forth in compliance with national regulations.

\section{Conclusion}

The answer to the question as to whether variations in IRB decisions can be completely avoided is probably no, but the answer to the question as to whether making decisions based on irrational or subjective criteria can be minimized, is yes. The aim for the development of EGES is to standardize the decision-making criteria according to internationally accepted guidelines to cover the basic ethical criterion. Going through the EGES would generate awareness about what IRB members have to consider before reaching their decision.

The EGES includes issues stated and agreed in international guidelines and regulations. However, it is a fact that national regulations may sometimes be more limiting than international guidelines due to religious, cultural or ethnic variations. Therefore, the IRB's willing to use the EGES as an evaluation sheet should revise it shortly to cover their national legislation. The revision of EGES to be in alliance with Turkish laws and regulations is in progress.

\section{References}

1. Ashcraft MH, Krause J. Social and Behavioral Researchers' Experiences With Their IRBs. Ethics \& Behavior, 2007; 17 (1): 1-17. doi. org/10.1080/10508420701309614.

2. Bledsoe CH et al. Regulating Creativity: Research and Survival in the IRB Iron Cage. Northwestern University Law Review, 2007; 101 (2): 593-641. doi.org/10.1080/13880290701347374.

3. Brown JG. Institutional Review Boards: A time for reform. Office of Inspector General, Department of Health and Human Services, 1998, OEI-01-97-00193.

4. Carlson RV, Boyd KM, Webb DJ. The revision of the Declaration of Helsinki: Past, present and future. Brit J Clin Pharmacol 2004; 57 (6): 695-713. doi.org/10.1111/j.1365-2125.2004.02103.x.

5. Cataloguing Williams Jr, J. Medical student holding a newborn Medical Ethics Manual. Biomedical Research - ethics Interprofessional Relations (Vol. 5). doi.org/Williams, J. R. (2015). Medical Ethics Manual (3rd ed). UK: WMA (World Medical Associatio). Retrieved from http://www. wma.net/en/30publications/30ethicsmanual/pdf/ethics_manual_en.pdf.

6. Catania JA et al. Survey of u.s. Human research protection organizations: workload and membership. J Empir Res Human Res Ethics: JERHRE, 2008. doi.org/10.1525/jer.2008.3.4.57. 
7. Cobb LA, Thomas GI, Dillard DH, Merendino KA, Bruce RA. An evaluation of internal-mammary-artery ligation by a double-blind technic. New Engl J Med 1959. doi.org/10.1056/NEJM195905282602204.

8. De Vries R, DeBruin DA, Goodgame A. Ethics Review of Social, Behavioral, and Economic Research: Where Should We Go from Here? Ethics and Behavior 2004.

9. Dekkers W, Boer G. Sham neurosurgery in patients with Parkinson's disease: is it morally acceptable? J Med Ethics 2001. doi.org/10.1136/ jme.27.3.151.

10. Elger BS, Caplan A. Consent and anonymization in research involving biobanks: differing terms and norms present serious barriers to an international framework. EMBO Reports 2006. doi.org/10.1038/ sj.embor.7400740.

11. Emanuel E, Wendler D, Grady C. What makes clinical research ethical? JAMA 2000; 283 (20): 2701-2711.

12. Emanuel EJ, Miller FG. The ethics of placebo-controlled trials - A middle ground. New Engl J Med 2001.doi.org/10.1056/ NEJM200109203451211.

13. Emanuel EJ et al. Oversight of human participants research: Identifying problems to evaluate reform proposals. Ann Intern Med 2004. doi. org/VL-141

14. Emanuel E, Wendler D, Killen J, Grady C. What makes clinical research in developing countries ethical? The benchmarks of ethical research. J Infect Dis 2004; 189: 930-937.

15. Emanuel EJ, Wendler D, Grady C. An ethical framework for biomedical research. In: Emanuel EJ, Grady C, Crouch RA, Lie RK, Miller FG, Wendler D (Eds). The Oxford textbook of clinical research ethics (pp. 123-133). New York: Oxford University Press, 2008.

16. Freeman T B, Vawter DE, Leaverton PE, Godbold JH, Hauser RA, Goetz CG, Olanow CW. Use of placebo surgery in controlled trials of a cellular-based therapy for Parkinson's disease. New Engl J Med 1999. doi.org/10.1056/NEJM199909233411311.

17. Green LA, Lowery JC, Kowalski CP, Wyszewianski L. Impact of institutional review board practice variation on observational health services research. Health Serv Res 2006; 41 (1): 214-230. doi.org/10.1111/ j.1475-6773.2005.00458.x.

18. Harayil MT. (2005). E r b r.

19. Horng S, Miller FG. Is Placebo Surgery Unethical? New Engl J Med 2002. doi.org/10.1056/NEJMsb021025.

20. Hostiuc S, Rentea I, Drima E, Negoi I. Placebo in Surgical Research: A Case-Based Ethical Analysis and Practical Consequences. BioMed Res Internat 2006. doi.org/10.1155/2016/2627181.
21. ICH Harmonised Tripartite Guideline. Guideline for good clinical practice E6(R1). ICH Harmonised Tripartite Guideline, 1996 (4): i-53. doi.org/10.1056/NEJMp1012246.

22. Keith-Spiegel P, Koocher GP. The IRB paradox: could the protectors also encourage deceit? Ethics Behav 2005; 15 (4): 339-349. doi. org/10.1207/s15327019eb1504_5.

23. Kim WO. Institutional review board (IRB) and ethical issues in clinical research Historical Views on Human Subject Research. Korean J Anesth 2012. doi.org/10.4097/kjae.2012.62.1.3.

24. London AJ, Kadane JB. Sham surgery and genuine standards of care: can the two be reconciled? Amer J Bioethics 2003; 3 (4): 61-64. doi. org/10.1162/152651603322614652.

25. Macklin R. After Helsinki: Unresolved issues in International Research. Kennedy Inst Ethics J 2001; 11 (1): 17-36.

26. McGuire AL, Beskow LM. Informed Consent in Genomics and Genetic Research. Ann Rev Genom Hum Genet 2010. doi.org/10.1146/annurev-genom-082509-141711.

27. Miller FG, Kaptchuk TJ. Sham procedures and the ethics of clinical trials. J Roy Soc Med 2004. doi.org/10.1258/jrsm.97.12.576.

28. Organizations I, Sciences M. International Ethical Guidelines for Epidemiological Studies. Biomed Res 2016.

29. Pritchard I. How do IRB members make decisions? A review and research agenda. J Empir Res Hum Res Ethics 2011; 6 (2): 31-46. doi. org/10.1525/jer.2011.6.2.31.

30. Standards and Operational Guidance for Ethics Review of HealthRelated Research with Human Participants. http://apps.who.int/iris/bitstr eam/10665/44783/1/9789241502948_eng.pdf?ua=1\&ua=1.

31. Stark L, Schrag ZM. Behind closed doors: IRBs and the Making of Ethical research (morality and society series). Sociol Res Online 2012; 17 (2). doi.org/10.1080/01947648.2013.768507.

32. The Center for Advanced Study Project Steering Committee. Improving the System for Protecting Human Subjects: Counteracting IRB "Mission Creep," 1-33. 2005. www.law.uiuc.edu/conferences/whitepaper/index.html.

33. Van Luijn HEM, Musschenga AW, Keus RB, Robinson WM, Aaronson NK. Assessment of the risk/benefit ratio of phase II cancer clinical trials by Institutional Review Board (IRB) members. Ann Oncol 2002; 13 (8): 1307-1313. doi.org/10.1093/annonc/mdf209.

34. Wolf BR, Buckwalter J. Randomized Surgical Trials and "Sham" Surgery: Relevance To Modern Orthopaedics and Minimally Invasive Surgery. Iowa Orthop J 2006.

35. World Medical Association (WMA). WMA Declaration of Helsinki - Ethical Principles 2013. 